\title{
Instantaneous Frequency Estimation of Multi-component Chirp Signals Based on Frft
}

\author{
Lili Zhang \\ College of Electronic Information Engineering \\ Shenyang Aerospace University \\ Shengyang, China
}

\author{
Sixin Liu, Junjun $\mathrm{Wu}$ \\ College of GeoExploration Science and Technology \\ Jilin University \\ Changchun,China
}

\begin{abstract}
For the problem of the instantaneous frequency estimation of multi-component chirp signals, a new method is presented. And the fractional Fourier transform (FrFT) method is applied to estimate instantaneous frequency of multi-component chirp signals. The chirp signal has the feature that different chirp signal can get the best energy concentration in its different fractional Fourier transform domain, so the multi-component chirp signals is firstly transformed through FrFT, and then we find the corresponding order $p$ of the every energy concentration and make the p order FrFT through a band-pass filter, and then it picks up each component. Finally the instantaneous frequency of each single-component chirp signal is estimated if each signal's wigner-ville distribution is found. The simulation results verify the availability of the proposed method.
\end{abstract}

Keywords-Instantaneous frequency estimation,Multicomponent chirp signals, Fractional Fourier transform (FrFT), Wigner-ville distribution

\section{INTRODUCTION}

The chirp signal is widely used in the areas of radar, communication, sonar, and earthquake. The instantaneous frequency estimate of the chirp signal has very important practical meaning and theoretical value. The instantaneous frequency estimate is interfered by multi-component cross term in processing a multi-component signal. The cross term can interfere the real signal. So it is difficult to analysis and explains the distributing of time-frequency. Some authors put forward many methods for restraining cross term effectively. Literature [1] combines Gabor transformation and WVD distribution, and it gains a new time-frequency distribution to restrain the interference of cross term. Literature [2] introduces a phase updating function to restrain the influences of self-cross term in a signal in base of WVD. These methods have achieved certain progress, but the cross term still exist. In this paper, a new method called FrFT(Fractional Fourier transform)is introduced. Fractional Fourier Transform is one of the tools used in the T-F analysis field. In the past decade the FrFT has attracted the attention of researchers in the optics and signal processing community, being a generalization of the classical Fourier transform.

In this paper, the method combines FrFT and WVD distribution in order to estimate the instantaneous frequency. first we get the FrFT of multi-component chirp signals ,and then it picks up each component using the feature that different chirp signal can get the best energy concentration in its different fractional Fourier transform domain. The point is to found the order p. Finally the instantaneous frequency of each single-component chirp signal is estimated according to the signal's Wigner Ville distribution. This method avoids the cross terms effect completely. Finally, the paper with two component chirp signals, for example, separated out each component and obtained the instantaneous frequency by using this method. The simulation results verify the availability of the proposed method.

\section{THE Fractional FOURIER TRANSFORMS THEORY}

The FrFT is a generalization of the conventional Fourier Transform and has a history in mathematical physics and in digital signal processing. Basically, it is a one-parameter subclass of the class of linear canonical transforms. This parameter is called the fractional order of the transform, usually denoted as $p$

Let $F$ denote the Fourier transform operation so that $X_{1}(\cdot)=F[x(t)]$ is the Fourier transform of the function $x(t)$.The $p$ th order fractional Fourier transform operation is denoted as $F^{p}$ so that $X_{p}(\cdot)=F^{p}[x(t)]$ is the $p$ th-order fractional Fourier transform of the function $x(t)$. $F^{1}$ corresponds to the ordinary Fourier operation $F$, $F^{0}$ and is the identity operation. $F^{2}$ corresponds to the coordinate reflection operation so that $F^{4}$ is also equivalent to the identity operation. We also have $F^{p 1} F^{p^{2}}=F^{p 1+p 2}$ the fractional Fourier transform can be defined for $0<|p|<2$ as [3]:

$$
X_{p}(u)=\int_{-\infty}^{+\infty} K_{p}(u, t) x(t) \mathrm{d} t
$$

Where

$K_{p}(u, t)=A_{\alpha} \exp \left[j \pi\left(u^{2} \cot \alpha-2 u t \csc \alpha+t^{2} \cot \alpha\right)\right]$ is the kernel function. $A_{a}=\sqrt{1-j \cot \alpha}, \quad \alpha=p \pi / 2, \quad p \neq 2 n, \mathrm{n}$ is the integer. The kernel $K_{p}(u, t)$ approaches $\delta(t-u)$ or $\delta(t+u)$ when $\mathrm{p}$ approaches 0 or $\mathrm{f} \pm 2$, respectively. The definition is easily extended outside the interval $[-2,2]$ by remembering that $F^{4}$ is the identity operation. The functions 
$X_{p}(u)$ for different values of a may be considered as different representations of the same signal $x(t)$.

$$
\begin{aligned}
& K_{p}(u, t) \\
& =\left\{\begin{array}{c}
\sqrt{\frac{1-j \cot \alpha}{2 \pi}} \exp \left(j\left(\frac{t^{2}+u^{2}}{2}\right) \cot \alpha-j u t \csc \alpha\right) \\
\text { if } \alpha \text { is not a multiple of } \pi \\
\delta(t-u) \\
\text { if } \alpha \text { is a multiple of } 2 \pi \\
\delta(t+u)
\end{array}\right. \\
& \text { if } \alpha+\pi \text { is a multiple of } 2 \pi \\
& =\sum_{n=0}^{\infty} e^{-j n \alpha} H_{n}(t) H_{n}(u)
\end{aligned}
$$

Where $\alpha$ indicates the rotation angle of transformed signal for the FrFT. $H_{n}(t)$ is the $n$ th-order normalized Hermite function with unit variance. The $n$ th-order normalized Hermite function with variance $\sigma$ is defined as

$$
H_{\sigma, n}(t)=\frac{1}{\left(2^{n} n ! \sqrt{\pi} \sigma\right)^{1 / 2}} h_{n}\left(\frac{t}{\sigma}\right) e^{-\left(t^{2} / 2 \sigma^{2}\right)}
$$

Where $h_{n}(\cdot)$ is the $n$ th-order Hermite polynomial. Because the normalized Hermite function with unitary variance, $H_{n}(\cdot)$ is the eigenfunction of the FrFT, (2) provides an eigendecomposition representation of the FRFT kernel. Using the kernel of the FrFT, the FrFT of the signal $x(t)$ by angle $\alpha$ is computed as

$$
\begin{aligned}
& X_{\alpha}(u)=\int_{-\infty}^{\infty} x(t) K_{\alpha}(t, u) d t \\
& =\sum_{n=0}^{\infty} H_{n}(u)\left(e^{-j n \alpha} \int_{-\infty}^{\infty} x(t) H_{n}(t) d t\right)
\end{aligned}
$$

Equation (4) indicates that the FRFT can be interpreted as a weighting summation of Hermite functions. The weighting coefficients are obtained from multiplying the phase term $e^{-j n \alpha}$ and the inner product of the input signal and the corresponding Hermite function. In [4], a rotation operation using Hermite functions has also been proposed, and the rotation output of the signal is also the weighting summation of Hermite functions. However the Hermite functions in [4] with a different scaling to those of the FrFT. Thus, it will have a different phase term in the weighting coefficients.

The $X_{-p}(u)$ is the inverse fractional Fourier transform.

That is

$$
x(t)=\int_{-\infty}^{+\infty} K_{-p}(u, t) X_{p}(u) \mathrm{du}
$$

Obviously, the FrFT transformation domain is a combination of the time and frequency domains. For angles $\alpha$ a close to $\alpha=\pi / 2$, for example for $\pi / 4 \leq|\alpha| \leq 3 \pi / 4$,we can consider the FrFT as the transformation being dominantly in the frequency domain. On the other hand, for small $\alpha,|\alpha|<\pi / 4$, the FrFT is dominantly in the time domain.

\section{THE FRFT CHARACTERISTIC OF THE CHIRP SigNAL}

Assuming a single-frequency chirp signal expression for

$$
y(t)=\exp \left(2 \pi j\left(f_{0} t+\frac{1}{2} k t^{2}\right)\right)
$$

The Wigner Ville distribution is $W(f, t)=\delta\left[f-\left(k t+f_{0}\right)\right]$

According to rotation invariant of a wigner-ville distribution, the projection of chirp signal in the fractional Fourier domain should be gathered at one point as long as the rotation angle of $\alpha$ and $\beta$ on the orthogonal[5], it is shown in fig. 1.The chirp signal has the feature that different chirp signal can get the best energy concentration in its different fractional Fourier transform domain, So we can rotate a different perspective (namely different order $\mathrm{p}$ )for the multi-component chirp signals in order to find the each energy gathering point. Most energy of each chirp signal is concentrated within a narrow band. So we make the $p$ order FrFT through a band-pass filter, and then each component is isolated. Finally the instantaneous frequency of each singlecomponent chirp signal is estimated if each signal's Wigner Ville distribution is found.

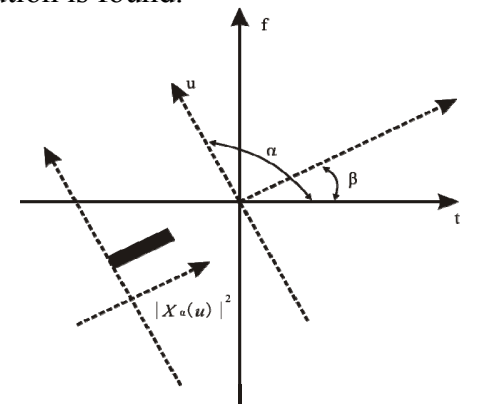

Figure 1. The FrFT and wigner-ville distribution of the chirp signals

\section{Simulation EXPERIMENT}

The practice effect of the algorithm stated in this paper is illuminated through the following simulation examples. The multi-component Chirp signals model is $x(t)=\exp \left(-j\left(15.8 t^{2}-5 t\right)\right)+\exp \left(-j\left(32.5 t^{2}-30 t\right)\right)$.fig. 2 is FrFT of this signal and the range of p order is [0,2],there are two peaks that can be seen from Fig. 2. They correspond to different order $\mathrm{p}$, namely $0.75,0.875$.Fig. 3 are the FrFT domain graphs when $p=0.75$ and $p=0.875$, then we make the data through a band-pass filter respectively. Each component is separated out. Fig. 4 is the Wigner Ville distribution graphs of each chirp signal. So the instantaneous frequency of each chirp signal is estimated. Fig. 5 shows the results. The method in this paper can eliminate the influence of noise. Fig. 6 is FrFT of this signal when $\mathrm{SNR}=5$, we find there are still two peaks that can be seen from Fig. 6. Fig. 7 are the FrFT domain graphs when $p=0.75$ and $p=0.875$ when 
$\mathrm{SNR}=5$, from the figure we can know the result have not changed although we add noise. So fig. 8 and fig. 9 are the same with the fig. 4 and fig. 5 .

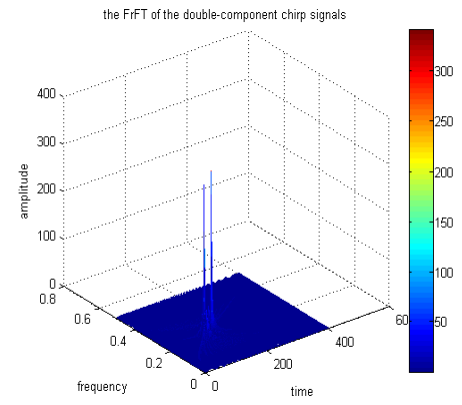

Figure 2. The FrFT of the chirp signals
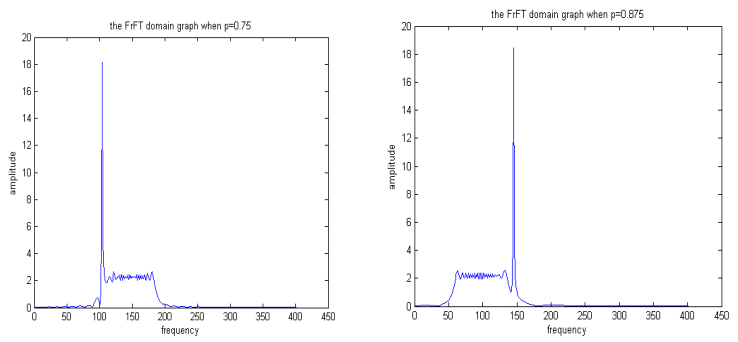

Figure 3. The FrFT of the chirp signals $(\mathrm{p}=0.75$ and $\mathrm{p}=0.875)$
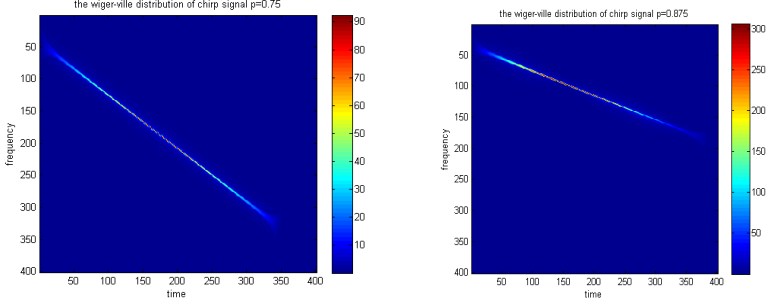

Figure 4. The wigner-ville distribution of each chirp signal
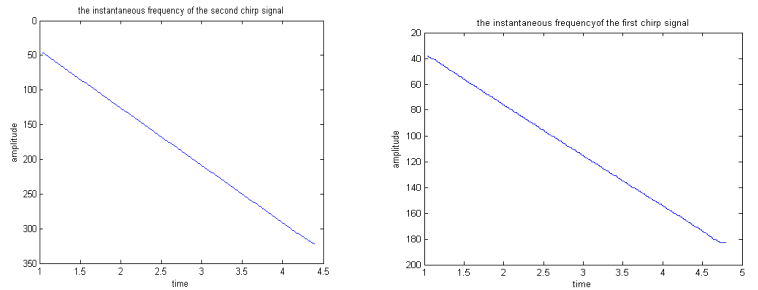

Figure 5. The instantaneous frequency estimation of each chirp signal

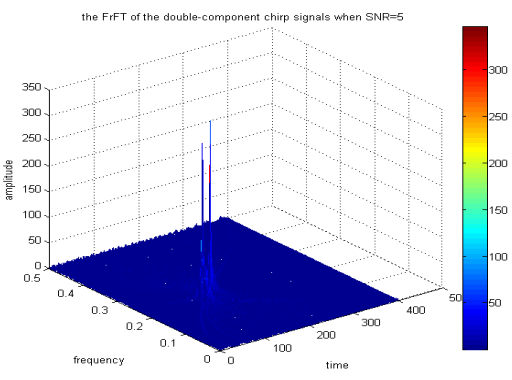

Figure 6. The FrFT of the chirp signals when $\mathrm{SNR}=5$
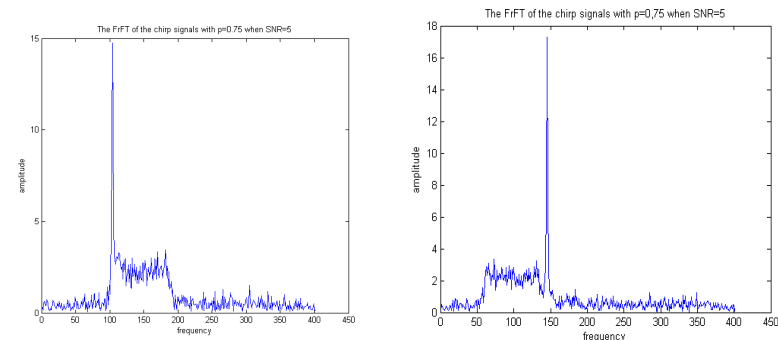

Figure 7. The FrFT of the chirp signals when $\mathrm{SNR}=5(\mathrm{p}=0.75$ and $\mathrm{p}=0.875$ )
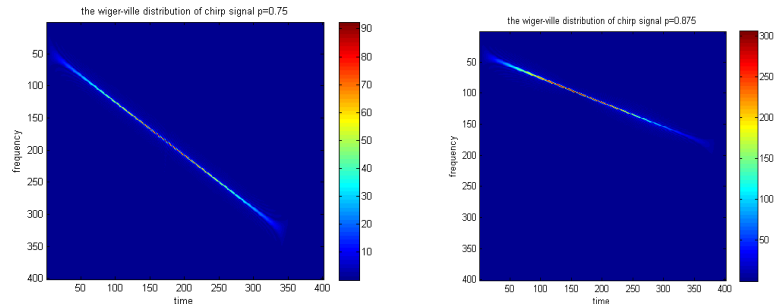

Figure 8. The wigner-ville distribution of each chirp signal when $\mathrm{SNR}=5$
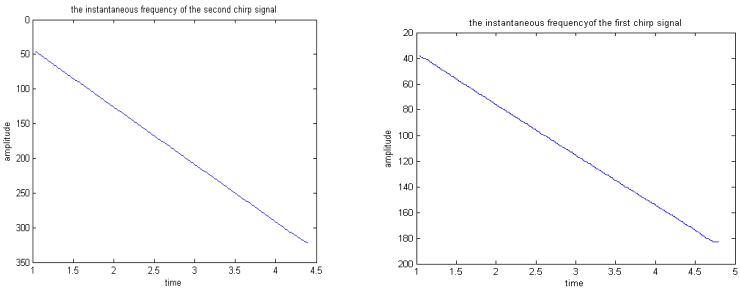

Figure 9. The instantaneous frequency estimation of each chirp signal when $\mathrm{SNR}=5$

\section{CONCLUTION}

Aiming at the estimation problem of instantaneous frequency of multi-component chirp signal, this paper puts forward a new estimation method. It solves the instantaneous frequency of multi-component chirp signal applying the fractional Fourier transform and Wigner Ville distribution. The chirp signal has the feature that different chirp signal can get the best energy concentration in its different fractional Fourier transform domain, so every chirp signal is separated 
through the fractional Fourier transform. Finally the instantaneous frequency is obtained by Wigner Ville distribution. This method is proved the efficiency through simulation experiment.

\section{ACKNOWLEDGMENT}

This research is supported by the National Natural

Science Foundation of China (Grant
No40874073\&41074076.).

\section{REFERENCES}

[1] ianu M , Isar A. The reduction of interference terms in the time frequency plane, Circuit s and Systems [J], 2003, pp.461-2464.

[2] WNG Yong, JIANG Yicheng .A new method for restrain the crossterms of time-frequency distributions. Chinese journal of electronics [J], 2008.vol:36, pp. 162-165.

[3] Haldun M. Ozaktas, Orhan Aytiir.Fractional Fourier domains. Signal Processing [J] 1995, vol: 46, pp119-124.

[4] B. A. Weisburn, T. W. Parks, and R. G. Shenoy, Separation of transientsignals, in Proc. 6th IEEE DSP Workshop, Oct. 1994, pp. 199-203.

[5] ANG xiuli,FENG xiaoping the parameter estimation and retrieval of the chirp signal based on fractional Fourier transform. Space electronic technology [J].2007 vol: 3, pp 78 82. 